\title{
Fingerprint Matching with Self Organizing Maps
}

\author{
Anastasia N. Ouzounoglou', Pantelis A. Asvestas² \\ and George K. Matsopoulos ${ }^{1}$ \\ ${ }^{1}$ School of Electrical and Computer Engineering \\ National Technical University of Athens \\ Greece \\ ${ }^{2}$ Department of Medical Instruments Technology \\ School of Technological Applications \\ Technological Educational Institute of Athens \\ Greece
}

\section{Introduction}

Fingerprint, namely the reproduction of a fingertip epidermis, produced when a finger is pressed against a smooth surface, is a human characteristic that has been systematically used for identification purposes for over 100 years.

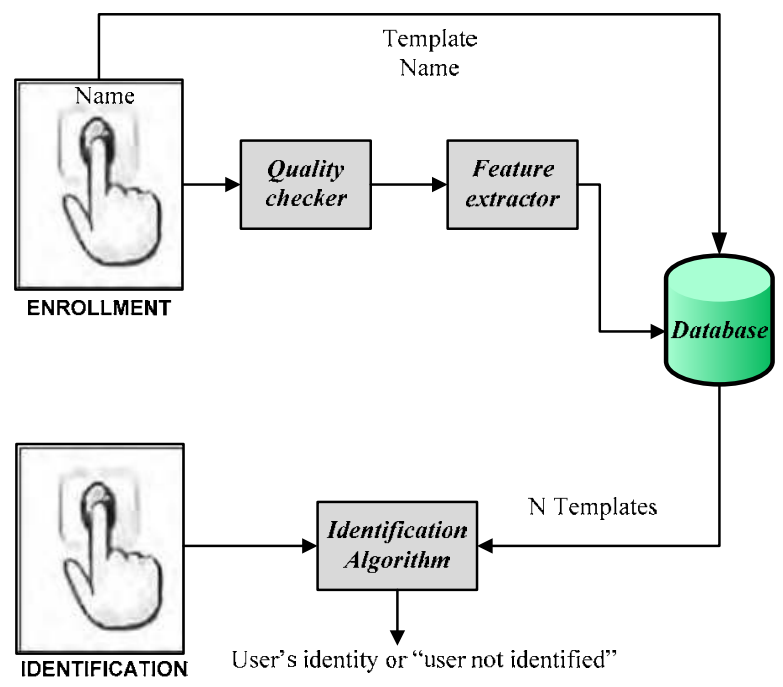

Fig. 1. Typical diagram of the fingerprint enrollment and identification processes. 
The identification involves the comparison, also known as fingerprint matching, of an input fingerprint image with a template image stored in a database and either the calculation of a matching score or the extraction of a binary decision (mated/non-mated) (Fig. 1).

Usually, a matching algorithm does not operate directly on grayscale fingerprint images but requires the derivation of an intermediate fingerprint representation by means of a feature extraction stage. The features that are used for fingerprint representation can be broadly categorized as follows (Yager \& Amin, 2004):

- features that are global characteristics of a fingerprint such as ridge flow

- features that refer to minutiae, such as ridge bifurcations and endings

- features that include all dimensional attributes of the ridge such as ridge path deviation, width, shape, pores, edge contours, breaks and scars.

The majority of the fingerprint identification systems are minutiae-based, but recently nonminutiae based systems as well as systems that use a combination of the features have been developed.

Jea et al, propose a fingerprint recognition system based only on minutiae matching (Jea \& Govindaraju, 2005). This method is satisfactory for partial fingerprint images, where the core points or other features cannot be fully estimated. However it produces poor results compared to other methods that make use of more features of the fingerprint. In (Chan et al, 2004), a fast verification method has been developed that is based on the matching of minutiae located in a region centered at a reference (core) point of the fingerprint. This method is fast enough since only minutiae in a subregion of the fingerprint image are used, although it is most of the times hard to calculate the reference point especially for low quality fingerprint images. Another minutiae-based method has been developed by (He et al, 2006). This method introduces minutiae and local ridge information in fingerprint representation and both features are used in the fingerprint verification process. (Gu et al, 2006) proposed a fingerprint matching technique that combines both the model - based orientation field and the minutiae. Jain et al made use of all three levels of fingerprint features in the fingerprint matching process (Jain et al, 2006). Furthermore, some trials have been conducted in order to cope with non-linear distortions in fingerprint matching. (Chen et al, 2006) addressed a fingerprint matching and verification method based on normalized fuzzy similarity measure. According to this method, the input and template fingerprint images are aligned used a minutiae-based method and then the similarity between the two images is assessed using a novel similarity measure based on fuzzy theory. (Ross et al,2006) used a thin-plate spline (TPS) function for the estimation of an "average" deformation model based on ridge curve correspondences for a specific finger, when several impressions of that finger are available.

Since the extraction of minutiae is a difficult and time consuming task (Jain et al, 1997), some trials have been made for the development of fingerprint registration systems without the use of minutiae (Jain et al, 2000; Liu et al, 2006). In (Jain et al, 2000), a reference point was detected and the fingerprint was tessellated around this point. Then, a feature vector was constructed using a bank of Gabor filters. This vector was called the "FingerCode". The matching between the two fingerprint images was conducted by estimating the Euclidean Distance between the FingerCodes. The drawback of this method is, as explained above, that the reference point is sometimes hard to be found and there are cases of destroyed or partial fingerprint images that cannot be detected at all. In the method proposed in (Liu et al, 2006), the orientation field of the fingerprint images was estimated initially and then the 
registration of the two images was carried out by maximization of the mutual information of the orientation fields. This method produced satisfactory results when the orientation field had been estimated in great detail, which is not always possible for low-quality fingerprint images.

This chapter proposes a minutiae-based fingerprint registration method. Initially, minutiae of the template image are extracted and validated. Then, the corresponding minutiae in the input image are determined by means of the proposed SOM-based algorithm. The obtained pairs of corresponding minutiae are used for the calculation of a matching score. The main advantages of the proposed method are that minutiae are extracted only on the template image and it is error-tolerant regarding the precise estimation of these points.

\section{Methodology}

In this chapter, an Automatic Fingerprint Identification Scheme (AFIS) is presented. The scheme comprises a series of processes as shown in the block diagram of Fig. 2. Without loss of generality, hereafter we denote the image of the fingerprint acquired during enrollment as the template $\left(I_{T}\right)$ and the representation of the fingerprint to be matched as the input image $\left(I_{\text {inp }}\right)$.

According to Fig. 2, the following processes are applied consequently only on the template image $I_{T}$ :

- $\quad$ Ridge Enhancement

- Binarization

- Background Removal

- $\quad$ Thinning

- Minutiae Extraction and Validation

- Minutiae Automatic Correspondence.

In the following analysis, these processes are described in more details:

\subsection{Ridge Enhancement}

The ridges of the template image $I_{T}$ (Fig. 3a) are enhanced using the method of oriented diffusion (Hastings, 2007), which is a recursive procedure. At each iteration $m$, the enhanced image, $I_{e n h}^{(m)}$, is obtained by means of the following equation:

$$
I_{e n h}^{(m)}(x, y)=I_{e n h}^{(m-1)}(x, y)+\gamma \frac{\partial^{2} I_{e n h}^{(m-1)}(x, y)}{\partial^{2} \theta}
$$

for $m=1,2, \ldots, M$, where $I_{e n h}^{(0)}=I_{T}, \theta=\theta(x, y)$ is the ridge orientation at pixel location $(x, y)$ and $\gamma$ is a constant. The ridge orientation of a fingerprint image, $I$, is estimated using the following procedure: initially the image is divided into blocks of size $w \times w$ and the gradients $\frac{\partial I(x, y)}{\partial x}=I_{x}(x, y)$ and $\frac{\partial I(x, y)}{\partial y}=I_{y}(x, y)$ are computed for each pixel $(x, y)$. The 
average gradient direction $\Phi(x, y)$ at each block centered at each pixel $(x, y)$ is estimated as follows (Bazen et al, 2007), (Pratt, 1989):

$$
\begin{aligned}
& V_{x}(x, y)=\sum_{u=x-\frac{w}{2}}^{x+\frac{w}{2}} \sum_{v=y-\frac{w}{2}}^{y+\frac{w}{2}} 2 I_{x}(u, v) I_{y}(u, v) \\
& V_{y}(x, y)=\sum_{u=x-\frac{w}{2}}^{x+\frac{w}{2}} \sum_{y=y-\frac{w}{2}}^{y+\frac{w}{2}}\left[I_{x}^{2}(u, v)-I_{y}^{2}(u, v)\right] \\
& \Phi(x, y)=\frac{1}{2} \tan ^{-1}\left(\frac{V_{y}(x, y)}{V_{x}(x, y)}\right)
\end{aligned}
$$

with $-\frac{\pi}{2}<\Phi(x, y) \leq \frac{\pi}{2}$.

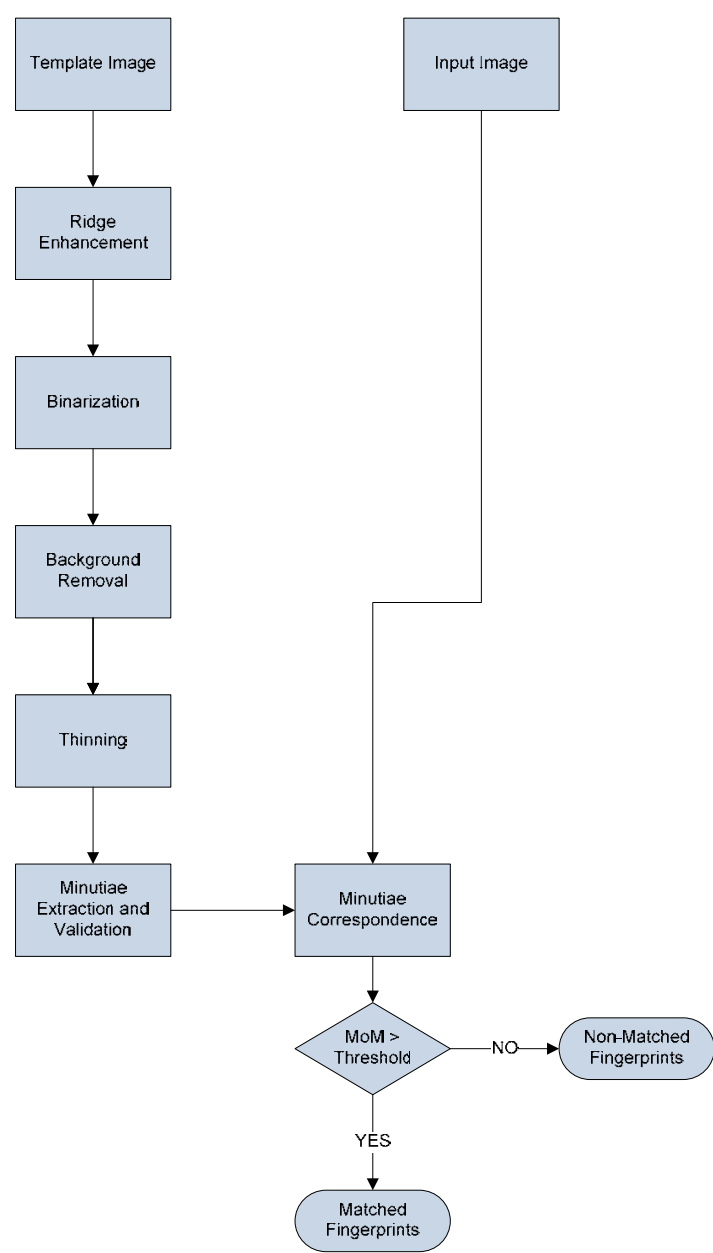

Fig. 2. Block diagram of the proposed automatic fingerprint identification scheme. 
Then, the orientation field $O(x, y)$, is defined as follows:

$$
O(x, y)=\left\{\begin{array}{lll}
\Phi(x, y)+\frac{1}{2} \pi & \text { for } & \Phi(x, y) \leq 0 \\
\Phi(x, y)-\frac{1}{2} \pi & \text { for } & \Phi(x, y)>0
\end{array}\right.
$$

with $-\frac{\pi}{2}<O(x, y) \leq \frac{\pi}{2}$.

Due to the presence of noise, the local ridge orientation may not always be correct. Thus, the orientation field is smoothed using a unit integral filter with size $w_{\Phi} \times w_{\Phi}$. Firstly, the orientation filed is converted into a continuous vector field: $O_{x}(x, y)=\cos (2 \cdot O(x, y)), O_{y}(x, y)=\sin (2 \cdot O(x, y))$, where $O_{x}(x, y), O_{y}(x, y)$ are the $x$ and $y$ components of the vector field, respectively. Then, the continuous vector field is filtered by applying a low-pass filter as follows:

$$
\begin{aligned}
& O_{x}^{\prime}(x, y)=\sum_{u=-w_{\Phi} / 2}^{w_{\Phi} / 2} \sum_{v=-w_{\Phi} / 2}^{w_{\Phi} / 2} H(u, v) \cdot O_{x}(x-u \cdot w, y-v \cdot w) \\
& O_{y}^{\prime}(x, y)=\sum_{u=-w_{\Phi} / 2}^{w_{\Phi} / 2} \sum_{v=-w_{\Phi} / 2}^{w_{\Phi} / 2} H(u, v) \cdot O_{y}(x-u \cdot w, y-v \cdot w)
\end{aligned}
$$

where $H$ is a two-dimensional low-pass filter with unit integral.

The smoothed orientation field of the fingerprint image at each pixel $(x, y)$ is computed using:

$$
\theta(x, y)=\frac{1}{2} \tan ^{-1}\left(\frac{O_{y}^{\prime}(x, y)}{O_{x}^{\prime}(x, y)}\right)
$$

The second derivative in (1) is calculated along an axis that forms an angle $\theta(x, y)$ with the $x$-axis using the following equation:

$$
\begin{aligned}
\frac{\partial^{2} I_{e n h}^{(m-1)}(x, y)}{\partial \theta^{2}}= & \frac{\partial^{2} I_{e n h}^{(m-1)}}{\partial x^{2}} \cdot \cos ^{2} \theta(x, y)+\frac{\partial^{2} I_{e n h}^{(m-1)}}{\partial y^{2}} \cdot \sin ^{2} \theta(x, y)+ \\
& 2 \frac{\partial^{2} I_{e n h}^{(m-1)}}{\partial x \partial y} \cdot \cos \theta(x, y) \cdot \sin \theta(x, y)
\end{aligned}
$$

The output of this step is denoted as $I_{\text {enh }}$ (Fig. 3b).

\subsection{Binarization}

For the binarization of the resulting enhanced template image, $I_{e n h}$, the second step of the method described in (Hastings, 2007) is applied. The second derivative of the filtered image 
is estimated in a direction normal to the orientation field. The second derivative is estimated using (6), but for $\theta^{\prime}=\theta+\frac{\pi}{2}$. The sign of the second derivative is then examined based on the fact that the second derivative of a function is negative in the area of a local maximum and positive in the area of a local minimum. The output of this step is denoted as $I_{B W}$ (Fig. 3c).

\subsection{Background Removal}

The first step for the estimation of the background of the binary image, $I_{B W}$, involves the partition of the image domain into blocks of $W \times W$ pixels. For each block, the mean value and the variance are calculated. If the variance is above a threshold, the pixels of this block are characterized as foreground pixels and the remaining ones as background pixel. Then only the foreground pixels of the binarized fingerprint image are maintained and the background pixels are set to the value 255. The output of this step is denoted as $I_{B R}$ (Fig. $3 d)$.

\subsection{Thinning}

The thinning process is applied on the negative version of $I_{B R}$. The two-subiteration thinning algorithm described in (Guo \& Hall, 1989) is applied in order to get a thinned version of the ridge fingerprint image. This algorithm uses a $3 \times 3$ thinning operator in the area of pixel $P$ as shown below:

$$
\begin{array}{lll}
P_{1} & P_{2} & P_{3} \\
P_{8} & P & P_{4} \\
P_{7} & P_{6} & P_{5}
\end{array}
$$

Let $C(P) \quad N(P), N_{1}(P)$ and $N_{2}(P)$ be defined as follows:

$$
\begin{gathered}
C(P)=\bar{P}_{2} \wedge\left(P_{3} \vee P_{4}\right)+\bar{P}_{4} \wedge\left(P_{5} \vee P_{6}\right)+\bar{P}_{6} \wedge\left(P_{7} \vee P_{8}\right)+\bar{P}_{8} \wedge\left(P_{1} \vee P_{2}\right) \\
N(P)=\min \left[N_{1}(P), N_{2}(P)\right] \\
N_{1}(P)=\left(P_{1} \vee P_{2}\right)+\left(P_{3} \vee P_{4}\right)+\left(P_{5} \vee P_{6}\right)+\left(P_{7} \vee P_{8}\right) \\
N_{2}(P)=\left(P_{2} \vee P_{3}\right)+\left(P_{4} \vee P_{5}\right)+\left(P_{6} \vee P_{7}\right)+\left(P_{8} \vee P_{1}\right)
\end{gathered}
$$

where the symbols, $\vee \wedge$ and ${ }^{-}$refer to logical OR, logical AND and logical complement, respectively.

A pixel that belongs to a ridge is removed from the ridge if the following three conditions are satisfied:

1. $C(P)=1$

2. $2 \leq N(P) \leq 3$

3. In odd iterations: $\left(P_{2} \vee P_{3} \vee \tilde{P}_{5}\right) \vee P_{4}=0$

In even iterations: $\left(P_{6} \vee P_{7} \vee \tilde{P}_{1}\right) \wedge P_{8}=0$ 
The algorithm stops when no new ridge pixels are deleted. The output of this step is denoted as $I_{T H}$ (Fig. 3e).

\subsection{Minutiae Extraction and Validation}

The minutiae extraction method used in this work is based on the calculation of the Crossing Number $(C N)$ (Maltoni et al, 2009). This method uses a $3 \times 3$ window in the local area of each ridge pixel $P$. The pixels in the area of $P$ are numbered as follows:

$$
\begin{array}{lll}
P_{4} & P_{3} & P_{2} \\
P_{5} & P & P_{9} \\
P_{6} & P_{7} & P_{8}
\end{array}
$$

The $C N$ for a ridge pixel $P$ is:

$$
C N=0.5 \sum_{i=1}^{8}\left|P_{i}-P_{i+1}\right|, \quad P_{9}=P_{1}
$$

If $\mathrm{CN}$ is one for a ridge pixel then the pixel is characterized as a ridge ending and if $\mathrm{CN}$ is three as a ridge bifurcation. However, the $C N$ algorithm gives rise to many false minutiae. Therefore, a minutiae validation algorithm is applied (Tico \& Kuosmanen, 2000). The algorithm uses an area of size $K \times K$ around each candidate minutiae. The central pixel of this area corresponds to the minutiae and is given the value -1 . The rest points are initialized with the value zero. The steps of validation for a candidate bifurcation point are as follows:

1. The three pixels that are connected to the bifurcation point are labeled with values $l=1$, 2 and 3 in a clockwise direction.

2. Three ridge branches, that originate from the three eight-connected to the minutiae pixels, are labeled with the corresponding value of $l$.

3. The number of transitions from 0 to 1,0 to 2 and 0 to 3 is counted in a clockwise direction along the border of the selected area. If all three transitions are equal to one then the point is characterized as a bifurcation point.

In Fig. 3f, the extracted minutiae of the template image are shown.

It must be noted that in this work only the minutiae that correspond to bifurcation points are used in the process of registration, while endings are not used at all. That is based on the fact that the number of bifurcation points of the images of the test database is from 14 to 25; that is an adequate number of neurons for the SOM algorithm. The number of ending minutiae points is from 32 to 59. If the ending points were to be used, the execution time of the algorithm would be larger without producing better results. Furthermore, SOM algorithm works better if the points are scattered in the image, something that is valid for bifurcation points. 


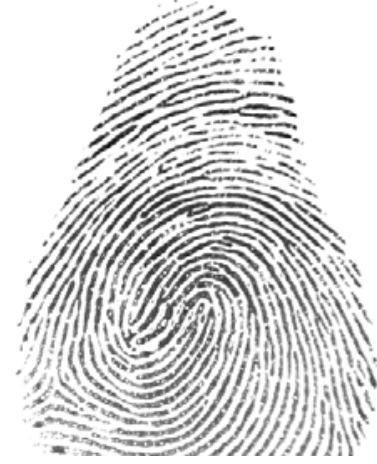

(a)

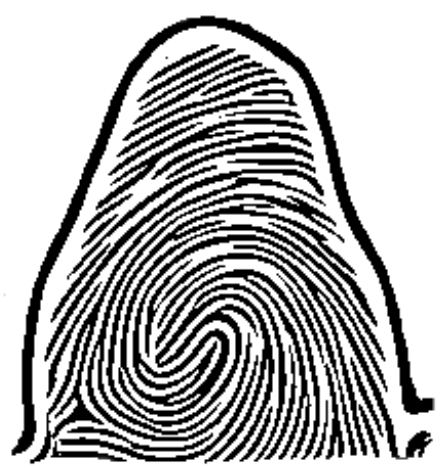

(c)

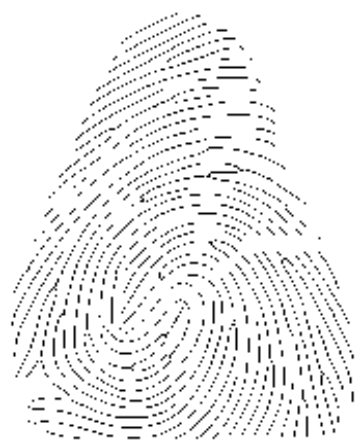

(e)

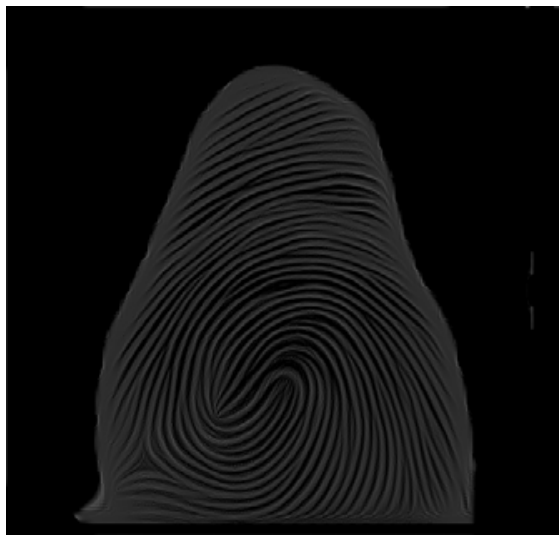

(b)

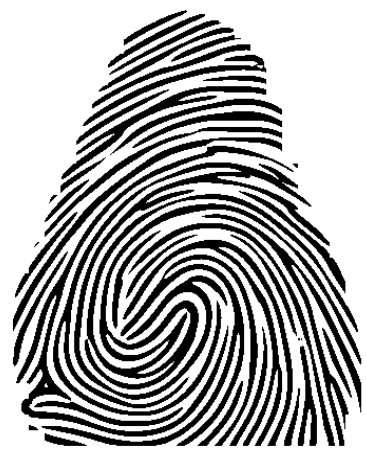

(d)

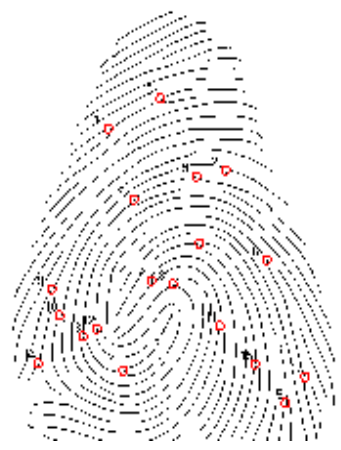

(f)

Fig. 3. Output images of the processes of the proposed automatic fingerprint identification scheme. (a) Template image. (b) Ridge enhanced image. (c) Binarized image. (d) Binarized image after background removal. (e) Thinned image. (f) Minutiae after the application of the extraction and validation process. 


\subsection{Minutiae Correspondence based on SOM algorithm}

An automatic method for establishing minutiae correspondences between the template image and the input image is applied based on the theory of the SOMs. The SOMs is a neural network algorithm, which uses a competitive learning technique to train itself in an unsupervised manner. Kohonen firstly established the relevant theory and explored possible applications (Kohonen, 2000). The Kohonen model comprises a layer of $N$ neurons, ordered usually in a one- or two-dimensional grid. The training of the network is performed in an iterative way. At each iteration $n$, an input vector $\mathbf{x} \in \mathfrak{R}^{m}$ is presented to the network; the neuron $j$ with weight vector $\mathbf{w}_{j} \in \mathfrak{R}^{m}$ is declared as the winning neuron, according to the following rule:

$$
j=\underset{i}{\arg \min }\left(\left\|\boldsymbol{x}-\boldsymbol{w}_{i}\right\|\right)
$$

The weight vectors of the winning neuron $j$ and its neighboring neurons $i$ are updated as follows:

$$
\mathbf{w}_{i}(n+1)=\mathbf{w}_{i}(n)+h_{i j}(n)\left[\mathbf{x}(n)-\mathbf{w}_{i}(n)\right]
$$

where $h_{i j}(n)=h\left(\left\|\mathbf{r}_{i}-\mathbf{r}_{j}\right\|, n\right)$ is a kernel defined on the neural network space as a function of the distance $\left\|\mathbf{r}_{i}-\mathbf{r}_{j}\right\|$ between neurons $i$ and $j$ and the iteration number $n$. This kernel is a Gaussian function, which has maximum value at inter-neuron distance in the case of $i=j$. The width of this function decreases monotonically with the iteration number. In this way, convergence to the global optimum is attempted during the early phases of the self-training process, whereas gradually the convergence becomes more local as the size of the kernel decreases.

Before proceeding to the analytical description of the network topology, some notations must be introduced. Let $\mu_{A}(I)=I(x, y),(x, y) \in A \subset Z^{2}$ denote the restriction of an image $I$ in the region $A$ and $\mathbf{T}_{\mathbf{w}}(A): Z^{2} \rightarrow \mathfrak{R}^{2}$ be a transformation with parameters $\mathbf{w}=\left(w_{1}, w_{2}, \ldots, w_{d}\right)$ of the region $A$, where $d$ is the number of parameters needed for the definition of the specific transformation. In this chapter, the similarity transformation was considered defined as follows:

$$
\begin{aligned}
& x^{\prime}=r \cos \theta \cdot x-r \sin \theta \cdot y+d x \\
& y^{\prime}=r \sin \theta \cdot x+r \cos \theta \cdot y+d y
\end{aligned}
$$

with parameter vector $\mathbf{w}=(r, d x, d y, \theta)$ and $d x, d y, r$ and $\theta$ is the horizontal displacement, the vertical displacement, the scaling and the angle of rotation, respectively. The value of each parameter of the used transformation is bounded according to the following inequalities:

$$
\begin{aligned}
& |d x| \leq d x_{\text {max }} \\
& |d y| \leq d y_{\text {max }} \\
& |\theta| \leq \theta_{\text {max }} \\
& |r| \leq r_{\text {max }}
\end{aligned}
$$


where $d x_{\max }, d y_{\max }, r_{\max }>0$ and $0<\theta_{\max }<\pi$. Furthermore, $\operatorname{MoM}\left(I_{1}, I_{2}\right)$ denotes a measure of match between two images $I_{1}$ and $I_{2}$. Without loss of generality, it is assumed that $\operatorname{MoM}\left(I_{1}, I_{2}\right)$ lies in the range $[0,1]$ where 1 indicates perfect matching and 0 indicates no matching.

Let $\mathbf{P}_{i}=\left(x_{i}, y_{i}\right),(i=1,2, \ldots, N)$ be the minutiae extracted from the template image, then the algorithm places a neuron on each one of them. Each neuron is associated with a square area $A_{i}=\left[x_{i}-R, x_{i}+R\right] \times\left[y_{i}-R, y_{i}+R\right]$ of $(2 R+1)^{2}$ pixels centered at the position of the neuron. Additionally, a weight vector $\mathbf{w}_{i}$, which holds the parameters of a local transformation (rigid or similarity), is assigned to each neuron.

The SOM network is trained as follows:

1 For each neuron, the components of the weight vector are initialized to default values (i.e. $w_{i}(0)=(0,0,0)$ for rigid transformation and $w_{i}(0)=(1,0,0,0)$ for similarity transformation) and the quantities $\operatorname{MoM}_{i}(0) \equiv \operatorname{MoM}\left(\mu_{A_{i}}\left(I_{T}\right), \mu_{\mathbf{T}_{w_{i}(0)}\left(A_{i}\right)}\left(I_{\text {inp }}\right)\right)$ are calculated, the variable $M o M_{\text {best }}$ is set to a very large (in magnitude) negative value and the iteration variable, $n$, is set to 1 .

2 While $n$ is less than $n_{\max }$ :

○ If the average value of $\operatorname{MoM}_{i}(n-1), \operatorname{MoM}_{\text {ave }}(n-1)=\frac{1}{N} \sum_{i={ }^{\prime}}^{N} \operatorname{MoM}_{i}(n-1)$, is better than $M o M_{b e s t}$, then $M o M_{b e s t}=\operatorname{MoM}_{\text {ave }}(n-1)$ and the current weights are stored as $\mathbf{w}_{i}(i=1,2, \ldots, N)$.

- An input vector, $\mathbf{s}(n)$, is generated pseudo-randomly.

○ For every neuron, the quantity $\operatorname{MoM}_{i}(n)=\operatorname{MoM}\left(\mu_{A_{i}}\left(I_{T}\right), \mu_{\mathbf{T}_{\mathbf{s}(n)}\left(A_{i}\right)}\left(I_{i n p}\right)\right)$ is calculated.

- The winning neuron, $k_{n}$, in the current iteration, is defined as $k_{n}=\underset{i}{\arg \max }\left(\operatorname{MoM}_{i}(n)\right)$ under the condition $\operatorname{MoM}_{k_{n}}(n)>\operatorname{MoM}_{\text {ave }}(n-1)$.

- The weights of the neurons are updated according to the following equation:

$$
\mathbf{w}_{i}(n)=\mathbf{w}_{i}(n-1)+h\left(k_{n}, n, i\right)\left[\mathbf{s}(n)-\mathbf{w}_{i}(n-1)\right]
$$

where $h\left(k_{n}, n, i\right)(i=1,2, \ldots . . N)$ is given by the following equation:

$$
h\left(k_{n}, n, i\right)=\left\{\begin{array}{c}
L^{n},\left\|\mathbf{P}_{k_{n}}-\mathbf{P}_{i}\right\|<a^{n} \cdot d_{0} \\
0, \quad \text { otherwise }
\end{array}\right.
$$

where $L, a, d_{0} \in \mathfrak{R}$ are parameters to be defined later and \|\| denotes the Euclidean norm.

- The iteration variable is increased by one. 
The best value of the measure of match, $M o M_{\text {best }}$, provides an index of the matching between the two images.

Several issues regarding the proposed method should be discussed. First of all, in order to cope with the differences in contrast and/or brightness between the template and the input image, the selected measure of match was the squared correlation coefficient, namely:

$$
\operatorname{MoM}\left(I_{1}, I_{2}\right)=\frac{\left(\sum_{x, y}\left[I_{1}(x, y)-\bar{I}_{1}\right]\left[I_{2}(x, y)-\bar{I}_{2}\right]\right)^{2}}{\sum_{x, y}\left[I_{1}(x, y)-\bar{I}_{1}\right]^{2} \sum_{x, y}\left[I_{2}(x, y)-\bar{I}_{2}\right]^{2}}
$$

where $\bar{I}_{1}$ and $\bar{I}_{2}$ are the mean pixel value for image $I_{1}$ and $I_{2}$, respectively.

As was mentioned before, a input vector $\mathbf{s}(n)$ is generated pseudorandomly, according to the following relation:

$$
\mathbf{s}(n)=\mathbf{w}_{k_{n}}+\mathbf{v}
$$

where $\boldsymbol{v}=\left(v_{1}, v_{2}, \ldots, v_{d}\right)$ is a $d$-dimensional normally distributed random variable with mean vector $\underbrace{(0,0, \ldots, 0)}_{d}$ and covariance matrix $\operatorname{diag}\left(\sigma_{1}^{2}(n), \sigma_{2}^{2}(n), \ldots, \sigma_{d}^{2}(n)\right)$. The standard deviation $\sigma_{j}(n)$ of the random variable $v_{j}(j=1,2, \ldots, d)$ varies with the iteration variable as follows:

$$
\sigma_{j}(n)=\left(U_{j}-L_{j}\right) \mathrm{e}^{-p n}
$$

where $U_{j}\left(L_{j}\right)$ denotes the maximum (minimum) allowed value for the $j$-th component of the input vector and $p$ determines the rate of exponential change of $\sigma_{j}(n)$. It must be noted that the above equations provide random signals which in general lie in the range $\left[w_{k_{n}, j}-\left(U_{j}-L_{j}\right), w_{k_{n}, j}+\left(U_{j}-L_{j}\right)\right]$. When a generated input vector is not in the allowed range $\left[L_{j}, U_{j}\right]$, then it is discarded and a new input vector is produced until $s_{j}(n) \in\left[L_{j}, U_{j}\right]$. The parameter $\sigma_{j}(n)$ controls how far from the weights of the current winning neuron the input vector can reach. As the iteration variable evolves, the magnitude of $\sigma_{j}(n)$ falls exponentially and the generated input signals are more localized around the weights of the current winning neuron. This is a desired property, since as the number of iterations grows, the weights of the current winning neuron get closer to the parameters of the solution of the registration problem.

The parameter $d_{0}$ provides the initial radius of a circular region around the winning neuron. Only neurons inside this region are updated. Usually, $d_{0}$ is set to the maximum distance between minutiae. As can be seen from (17), this distance is reduced with geometric rate determined by the parameter $a(0<a \leq 1)$. The parameter $L$ acts like a gain constant for the magnitude of the update that is applied to the weights of the neurons. This parameter also 
decreases geometrically as the iteration variable evolves. The range of values $L$ is between 0.99 and 1.0 .

It should be pointed out that a sufficient number of minutiae should be extracted in order to achieve an accurate registration result. Furthermore, the minutiae must be distributed over the whole image (if possible). The degree of sparseness of the bifurcation points can be determined by checking if the standard deviation of the $x-y$ coordinates is above a predefined threshold. Experiments have shown that for fingerprint images twelve minutiae, with standard deviation of the $x-y$ coordinates that exceeds 100, are sufficient in order to obtain accurate registration results. Since the transformed region $\mathbf{T}_{\mathbf{s}(n)}\left(A_{i}\right)$ does not have integer coordinates, bilinear interpolation (Press et al, 1992) is used in order to calculate $\operatorname{MoM}_{i}(n)$.

\section{Results}

In order to assess the performance of the proposed automatic fingerprint identification scheme, the VeriFinger_Sample_DB database of fingerprint images was used (Neurotechnology, 2007). The database contains fingerprint images from nine different persons, for six fingers of each person and for eight impressions of each finger; thus 432 images in total. Each fingerprint image has size $504 \times 480$ pixels. Two different data sets were used. The first data set (SET I) consists of fingerprint images subject to known transformations, while the second one (SET II) comprises of fingerprint image pairs from the database (unknown transformation).

\subsection{SET I: Data subject to known transformations}

The data of SET I consist of 11 fingerprint images from the VeriFinger_Sample_DB database. Each image is considered as a template image and is transformed into two different input images according to two affine transformations (Affine-1, Affine-2); thus, a total of 22 image pairs were finally obtained for further testing. The affine transformation function is described as follows:

$$
\begin{aligned}
& X=s \cos \theta\left(x-x_{c m}\right)-s \sin \theta\left(y-y_{c m}\right)+t_{1}+x_{c m} \\
& Y=s \sin \theta\left(x-x_{c m}\right)+s \cos \theta\left(y-y_{c m}\right)+t_{2}+y_{c m}
\end{aligned}
$$

where $x(X), y(Y)$ are the column and row indices, respectively, in the template (input) image and $\left(x_{c m}, y_{c m}\right)$ are the coordinates of the geometric centre of the template image, $s$ is the scaling parameter, $\theta$ the rotation angle and $t_{1}, t_{2}$ the translation along $x$-axis and $y$-axis, respectively. For Affine-1, the parameters used were: $s=1, \theta=5^{\circ}, t_{1}=5$ and $t_{2}=5$, while for Affine-2: $s=1.05, \theta=10^{\circ}, t_{1}=10$ and $t_{2}=10$.

After the sequential application of the processes described in Fig. 2, the number of the extracted minutiae in the template images of the data SET I varied from 11 to 30 points. The actual corresponding points, $\mathbf{Q}_{i}$, in the input image of each pair were obtained by transforming the minutiae of the template image according to (21). 
The performance of the proposed automatic point correspondence detection algorithm was quantitatively assessed using the Root Mean Square Error (RMSE). The RMSE was calculated between the detected points $\tilde{\mathbf{Q}}_{i}$ and the actual corresponding points $\mathbf{Q}_{i}$, $i=1,2, \ldots, N$ according to the equation:

$$
R M S E=\sqrt{\frac{1}{N} \sum_{i=1}^{N}\left\|\mathbf{Q}_{i}-\tilde{\mathbf{Q}}_{i}\right\|^{2}}
$$

The implementation of the algorithm described in the previous section assumes that a number of parameters are beforehand determined. The values of all parameters used are listed in Table 1. The same values of the parameters were applied to all images. It must be also noted that the proposed SOM algorithm was executed ten times for each image pair and the average value of the RMSE (in pixels) was calculated.

\begin{tabular}{|c|c|c|c|}
\hline Equation & Description & Symbol & Value \\
\hline$(1)$ & $\begin{array}{c}\text { Iterations of Oriented Diffusion (Ridge } \\
\text { Enhancement) }\end{array}$ & M & 100 \\
\hline$(1)$ & Spread of Gaussian Filter (Ridge Enhancement) & $r$ & 2 \\
\hline$(2)$ & Block Size for Orientation Field & $w \times w$ & $16 \times 16$ \\
\hline \multirow[t]{3}{*}{ (4) } & Size of Unit Integral Filter (Orientation Field) & $w_{\Phi} \times w_{\Phi}$ & $3 \times 3$ \\
\hline & Block Size for Image Segmentation & $W \times W$ & $15 \times 15$ \\
\hline & Area Size for Minutiae Validation & $K \times K$ & $23 \times 23$ \\
\hline \multicolumn{4}{|c|}{ SOM Algorithm Parameters } \\
\hline$(17)$ & Initial Neighbourhood Size of Winning Neuron & $d_{0}$ & $\begin{array}{c}\text { max distance } \\
\text { between neurons }\end{array}$ \\
\hline (17) & Rate of Change of $d_{0}$ & $a$ & 0.90 \\
\hline (17) & $\begin{array}{l}\text { Gain constant for the magnitude of the update that } \\
\text { is applied to the weights of the neurons - Learning } \\
\text { Rate }\end{array}$ & $L$ & 0.995 \\
\hline \multirow[t]{7}{*}{$(20)$} & Rate of change of input vector range & $p$ & 0.01 \\
\hline & Half Size of Square Region of each neuron & $R$ & 10 \\
\hline & Number of Iterations & $n_{\max }$ & 6,000 \\
\hline & Maximum Value of Scaling & $r_{\max }$ & 1.4 \\
\hline & $\begin{array}{c}\text { Maximum Value of Horizontal Displacement (in } \\
\text { pixels) }\end{array}$ & $d x_{\max }$ & 100 \\
\hline & $\begin{array}{c}\text { Maximum Value of Vertical Displacement (in } \\
\text { pixels) }\end{array}$ & $d y_{\max }$ & 100 \\
\hline & Maximum Value of Angle of Rotation (degrees) & $\theta_{\max }$ & 20 \\
\hline
\end{tabular}

Table 1. Implementation parameters of the proposed automatic fingerprint identification scheme.

An example of the performance of the proposed algorithm in defining automatic correspondence is shown in Fig. 4 for typical fingerprint images of SET I. A template image 
of the SET I is displayed in Fig. 4a along with its input images as transformed by the Affine-1 (Fig. 4c) and Affine -2 (Fig. 4e) transformations. The minutiae of the template thinned image are shown in Fig. $4 \mathrm{~b}$ as red dots. The actual corresponding points, calculated by the Affine-1 (Fig 4d) and Affine-2 (Fig 4f) transformations of the minutiae in the template image according to (18), are marked with red dots, while the corresponding points detected by the SOM algorithm are marked with yellow dots in the same figures. From this figure, it is evident that the detected points by the proposed algorithm correctly match the actual corresponding points.

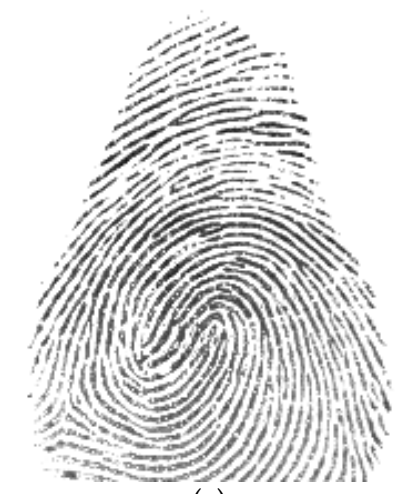

(a)

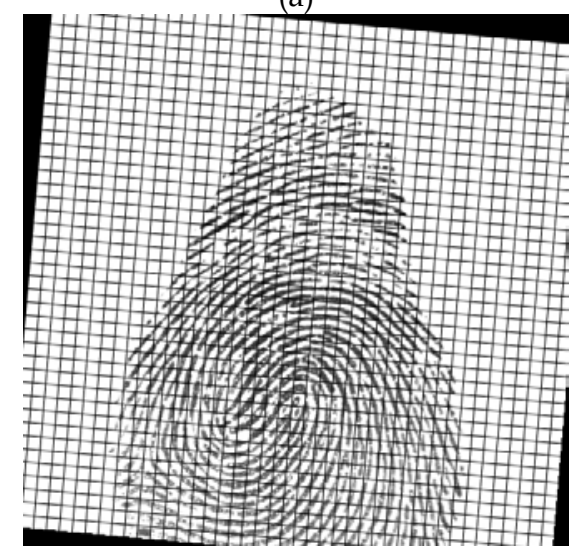

(c)

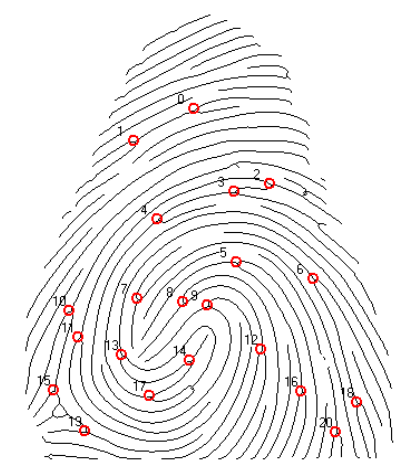

(b)

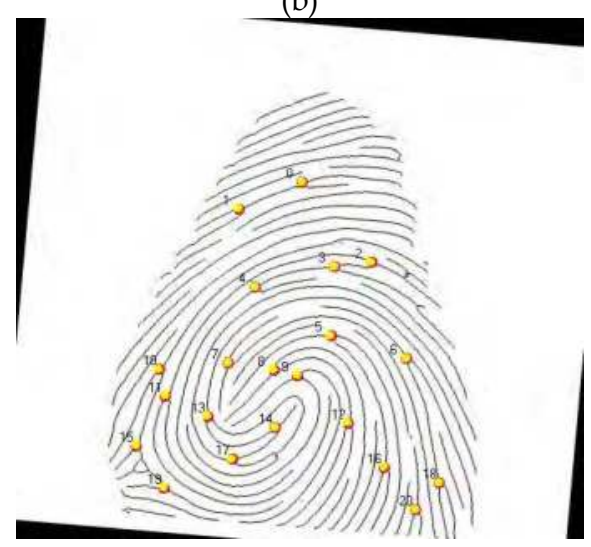

(d) 


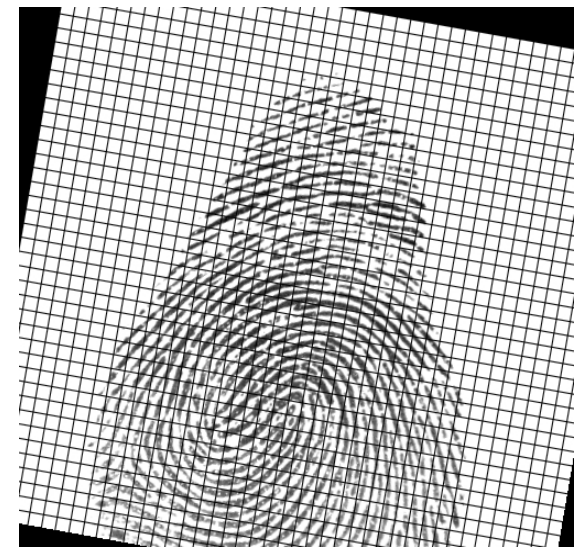

(e)

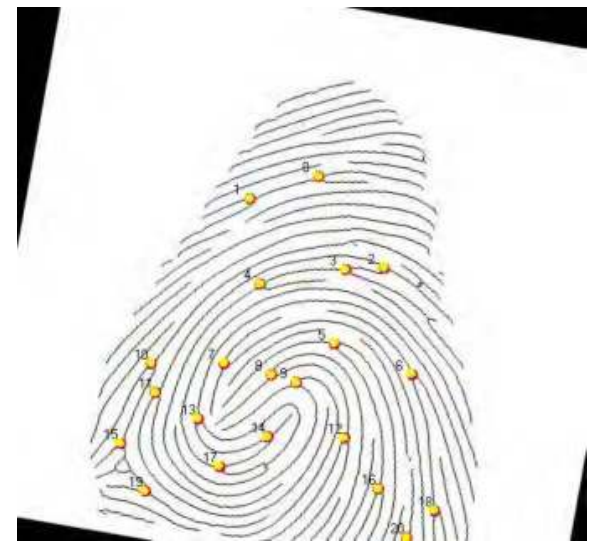

(f)

Fig. 4. The performance of the proposed SOM algorithm in defining automatic point correspondence of a typical fingerprint image of SET I and its transformed images. Red dots indicate the actual corresponding points, while yellow dots indicate the detected points using the SOM algorithm. (a) Typical fingerprint template image. (b) Minutiae of the template image, after the application of the extraction and validation process. (c) Transformed template image using the Affine-1 transformation. (d) Minutiae correspondences of the template and Affine- 1 transformed image using the SOM algorithm. (e) Transformed template image using the Affine-2 transformation. (f) Minutiae correspondences of the template and Affine- 2 transformed image using the SOM algorithm.

Furthermore, the performance of the proposed SOM algorithm is tested for a typical fingerprint image of SET I and its transformed image (Affine-1 transformation) against the number of iterations. Fig. 5(a) depicts the initial position of the minutiae in the input image (before the training process), while Fig. 5(b)-(d) show three different phases of the network training, after $800,2,800$ and 6,000 iterations, respectively. A plot of the average RMSE, as well as of the best values of the MoM, between the detected points and the actual corresponding points with respect to the number of iteration is shown in the diagram of Fig. 5(e). As the number of iteration increases, the value of the average RMSE drops from its initial value of 11.267 pixels to the value of 5.197 pixels, after 800 iterations (Fig.5(b)) and to the value of 0.581 pixels after 2,800 iterations (Fig.5(c)). The value of the MoM increases from its initial value to the value of 0.11 after 800 iterations and to the value of 0.32 after 2,800 iterations. The average RMSE gets its best value ( 0.389 pixels) after 3,000 iterations (Fig.5(d)) and then it remains stable (up to 10,000 iterations), while the average MoM gets its best value of 0.94 after 6,000 iterations.

The performance of the SOM algorithm in terms of the average RMSE was also studied for different values of two important parameters of the algorithm: the parameter $L$ and the parameter $a$ for a typical image pair of SET I, as transformed by the Affine-1. The parameter $L$ acts like a gain constant for the magnitude of the update that is applied to the weights of the neurons and the range of its value was between 0.99 and 1.0. The parameter $a$ relates with the reduction rate of the distance of the initial radius around the winning neuron and the range of its values was between 0.1 and 1.0. During these experiments, all the other parameters of the SOM algorithm were set to values included in Table 1. As can be noticed 
from Fig. 6a, the performance of the SOM algorithm is relative stable for different values of $L$, with lowest value of the average RMSE for $L=0.995$. Similarly, from Fig. $6 \mathrm{~b}$, the best performance of the SOM algorithm in terms of average RMSE was obtained for $a=0.90$.

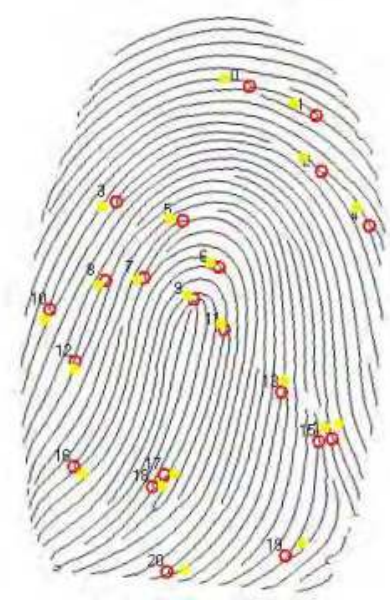

(a)

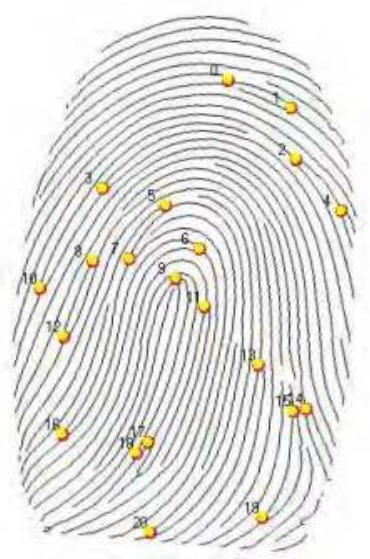

(c)

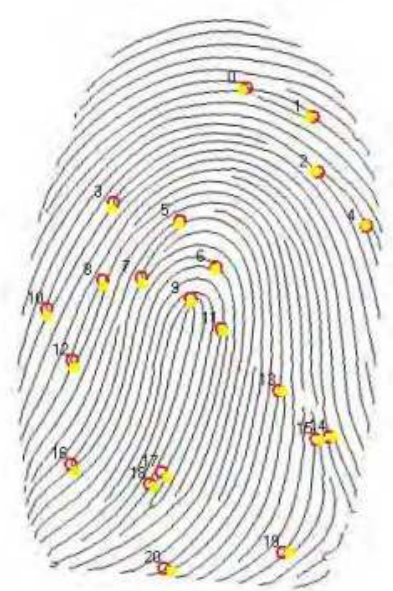

(b)

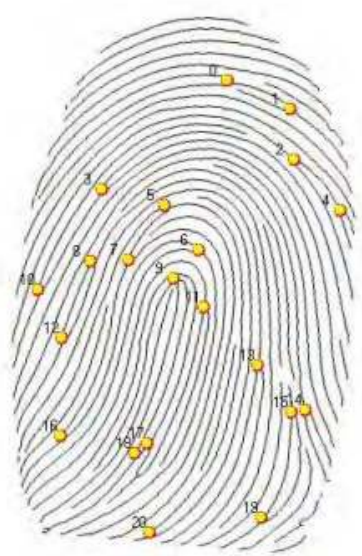

(d) 


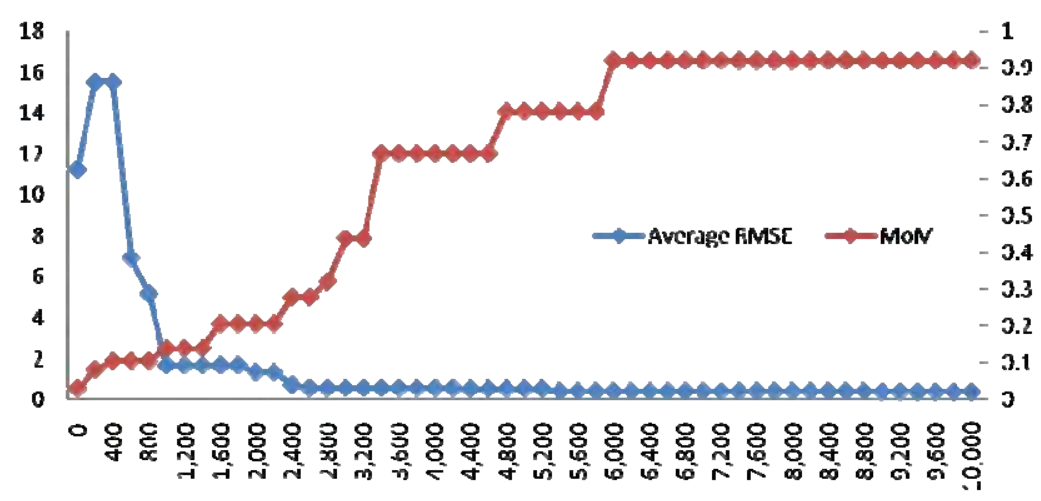

Nunbero: iterations

(e)

Fig. 5. The performance of the proposed SOM algorithm in defining automatic point correspondence for a typical fingerprint image of SET I and its transformed image (Affine-1 transformation) against the number of iterations. Red dots indicate the actual corresponding points, while yellow dots indicate the detected points using the SOM algorithm. (a) Initial points position. (b)-(d) Correspondences obtained after 800, 2,800 and 6,000 iterations, respectively. (e) Performance of the SOM algorithm in terms of the average RMSE and the best value of MoM for the specific pair with respect to the number of iterations.

Quantitative results from the application of the proposed automatic fingerprint identification scheme to SET I are presented in Table 2 in terms of the best values of MoM and the average RMSE (in pixels). For the Affine- 1 transformation, the average RMSE lies between 0.389 and 0.478 pixels. For the Affine- 2 transformation, the average RMSE lies between 0.643 and 2.089 pixels.

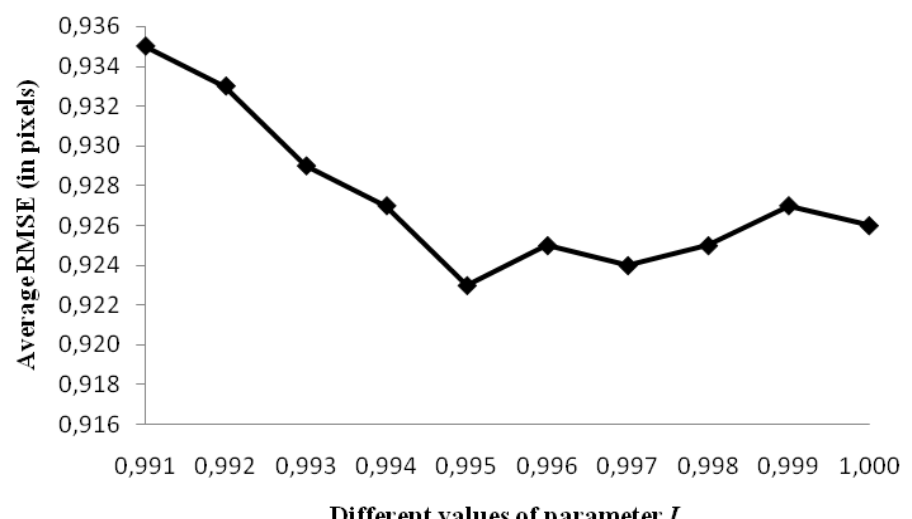

(a) 


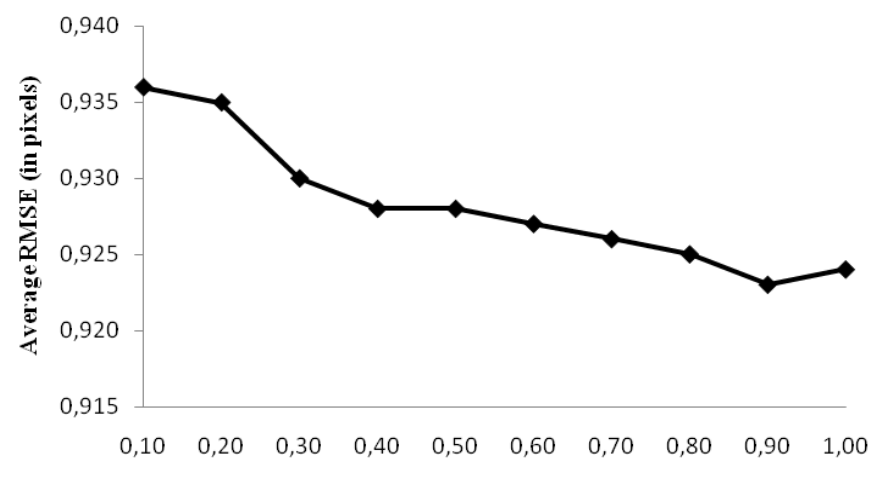

Different values of parameter $a$

(b)

Fig. 6. The performance of the proposed SOM algorithm in defining automatic point correspondence for a typical fingerprint image of SET I and its transformed image (Affine-1 transformation) against (a) different values of the parameter $L$ and (b) different values of the parameter $a$.

From Table 2, it is evident that the proposed algorithm is capable of determining correct correspondences with subpixel accuracy for the Affine-1 transformation. However, as is expected, its performance depends on the kind of transformation applied to the image pairs. Thus, the value of the average RMSE deteriorates for Affine-2 transformation. Yet again, the best values of the RMSE for the transformations remain relatively low, while the low values of the standard deviation indicate high reproducibility of the proposed algorithm for all transformations used.

\begin{tabular}{|c|c|c|c|c|}
\hline \multirow{2}{*}{$\begin{array}{c}\text { Pairs of SET } \\
\text { I }\end{array}$} & \multicolumn{2}{|c|}{ Affine-1 transformation } & \multicolumn{2}{c|}{ Affine-2 transformation } \\
\cline { 2 - 5 } & Average RMSE & MoM & Average RMSE & MoM \\
\hline 1 & & & & \\
\hline 2 & 0.389 & 0.943 & 0.643 & 0.831 \\
\hline 4 & 0.462 & 0.949 & 0.6999 & 0.863 \\
\hline 5 & 0.448 & 0.941 & 1.317 & 0.689 \\
\hline 6 & 0.478 & 0.945 & 0.746 & 0.679 \\
\hline 7 & 0.471 & 0.919 & 1.805 & 0.562 \\
\hline 8 & 0.448 & 0.897 & 2.089 & 0.540 \\
\hline 9 & 0.437 & 0.912 & 1.414 & 0.463 \\
\hline 10 & 0.390 & 0.954 & 0.805 & 0.729 \\
\hline 11 & 0.439 & 0.950 & 0.808 & 0.791 \\
\hline Mean & 0.404 & 0.912 & 0.722 & 0.805 \\
\hline STD & $\mathbf{0 . 4 3 6}$ & $\mathbf{0 . 9 3 1}$ & $\mathbf{1 . 0 7 7}$ & $\mathbf{0 . 7 0 1}$ \\
\hline $\mathbf{0 . 0 3 0 7}$ & $\mathbf{0 . 0 1 9}$ & $\mathbf{0 . 5 0 1}$ & $\mathbf{0 . 1 3 0}$ \\
\hline
\end{tabular}

Table 2. Quantitative results obtained by the proposed automatic fingerprint identification scheme to SET I in terms of best value of MOM and the average RMSE (in pixels). 


\subsection{SET II: Data subject to unknown transformations}

The SET II comprises of all the fingerprint images of the VeriFinger_Sample DB (Neurotechnology, 2007). This database consists of 408 fingerprint images. Tests have been carried out on 1,428 image pairs of same fingers and on 1,428 image pairs of different fingers. Each pair consists of a template and an input image, and the transformation that associates these two images is, unlike the previous case, unknown.

The proposed automatic fingerprint identification scheme was applied to all image pairs using the same values of the various parameters listed in Table 1 . Since the actual corresponding points $\mathbf{Q}_{i}, i=1,2, \ldots, N$ cannot be directly calculated by transforming the minutiae of the template image, a different procedure for the visual evaluation of the proposed algorithm had to be applied. Initially, minutiae were extracted in both the template and input images, according to the aforementioned procedures. Then, new minutiae of the input image were obtained by the application of the SOM algorithm. These two sets of minutiae on the input image were visually compared.

In Fig. 7, the performance of the proposed automatic fingerprint identification scheme, including the SOM algorithm, is visually assessed for a fingerprint image pair of SET II. In Fig. $7 \mathrm{a}$ and $7 \mathrm{~b}$, the initial template image of a finger and the input image of the same figure, as obtained with different settings, are displayed. Fig. 7c displays the thinned input image along with its extracted minutiae (red dots) and the minutiae (yellow dots) as obtained by the application of the proposed scheme. It can be noticed that a satisfactory correspondence has been achieved for the majority of the minutiae. For this particular pair, the best value of MoM was 0.798 .

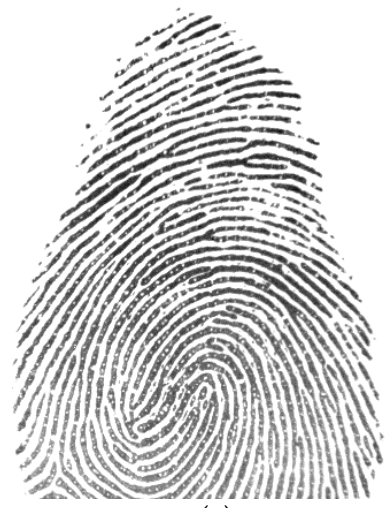

(a)

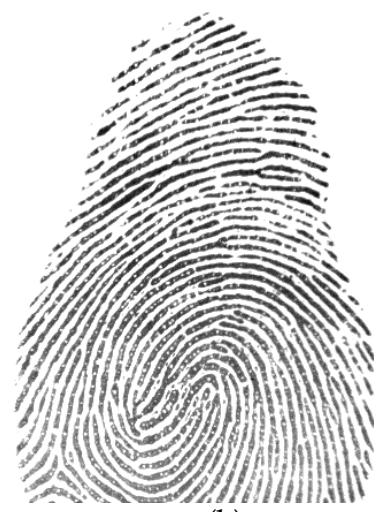

(b) 


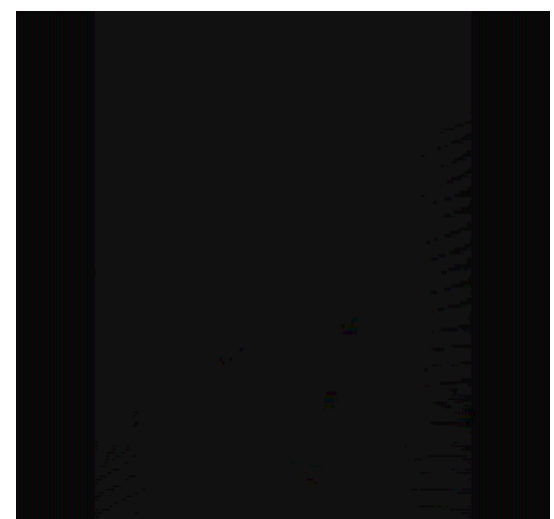

(c)

Fig. 7. The performance of the proposed SOM algorithm in defining automatic point correspondence for a fingerprint image pair of SET II (same finger under different acquisition settings). (a) Initial template image. (b) The input image. (c) Thinned input image along with its extracted minutiae (red dots) and the estimated minutiae as obtained by the proposed scheme (yellow dots).

In the case of applying the proposed automatic fingerprint identification scheme in pairs of different fingers, the results are considerably deteriorated, as it was expected. In Fig. 8a and $8 \mathrm{~b}$, two fingerprint images of different fingers are displayed. Fig. 8c shows the thinned image along with its extracted minutiae (red dots) and the minutiae (yellow dots), as obtained by the application of the proposed scheme, where it is evident the failure of minutiae correspondence. For this particular example, the best value of MOM was 0.145 .

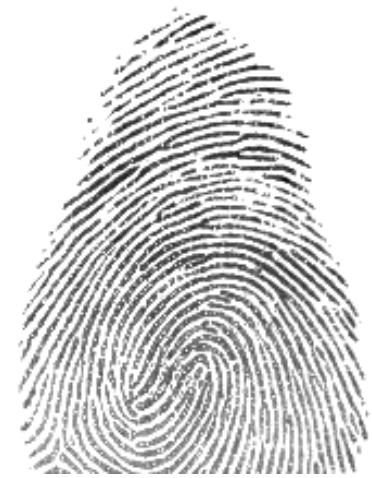

(a)

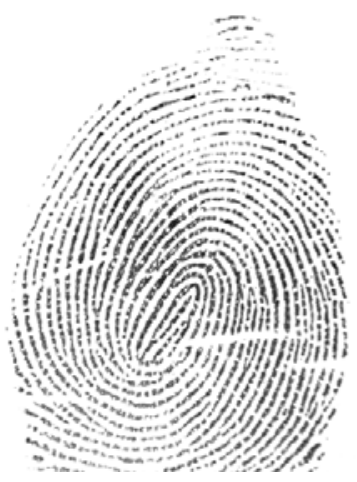

(b) 


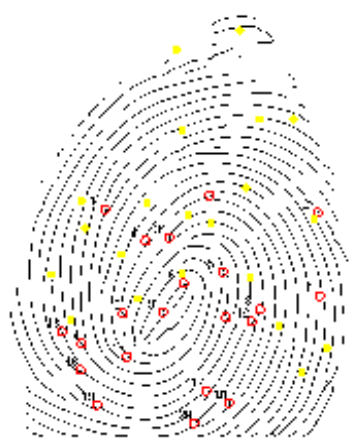

(c)

Fig. 8. The performance of the proposed SOM algorithm in defining automatic point correspondence for a fingerprint image pair of SET II (same finger of different person under different acquisition settings). (a) Initial template image. (b) The input image. (c) Thinned input image along with its extracted minutiae (red dots) and the estimated minutiae as obtained by the proposed scheme (yellow dots).

Different types of error rates are used as metrics for the operative capability of biometric authentication systems in general and for fingerprint image identification systems in particular. The result of a comparison in the feature matcher within a fingerprint image recognition system is called Matching Score. It measures the similarity between the fingerprint image and the stored template. If the value of Matching Score approaches 1 (if normalized between in the range [0,1]), the more likely that both fingerprints originate from the same finger. On the other hand, if Matching Score is near 0, it will be quite probable that both fingerprints are from different fingers.

The decision of the system is determined by threshold T, i.e. if Matching Score passed the threshold, the fingerprints are regarded as being of the same finger (matching pair) whereas if Matching Score is below the threshold, the fingerprints are regarded as being different (non-matching pair). The performance of a biometric system is assessed by means of the False Acceptance Rate (FAR), False Rejection Rate (FRR) and Equal Error Rate (EER) for various values of the threshold:

$$
\begin{aligned}
& F A R(T)=\frac{\text { Number of comparisons of different fingers resulting in a match }}{\text { Total number of different fingers }} \\
& \operatorname{FRR}(T)=\frac{\text { Number of comparisons of the same fingers resulting in a non-match }}{\text { Total number of the same fingers }} \\
& E R R=F A R\left(T^{*}\right)
\end{aligned}
$$

where $T^{*}$ is the value of the threshold, such that $\operatorname{FAR}\left(T^{*}\right)=\operatorname{FRR}\left(T^{*}\right)$.

The FAR and FRR were calculated by thresholding the best MoM obtained by the SOMbased algorithm for the data of SET II. The corresponding curves are shown in Fig. 9. The 
intersection point of the two curves corresponds to the ERR. The value of the ERR is 0.08 and obtained for $T^{*}=0.219$.

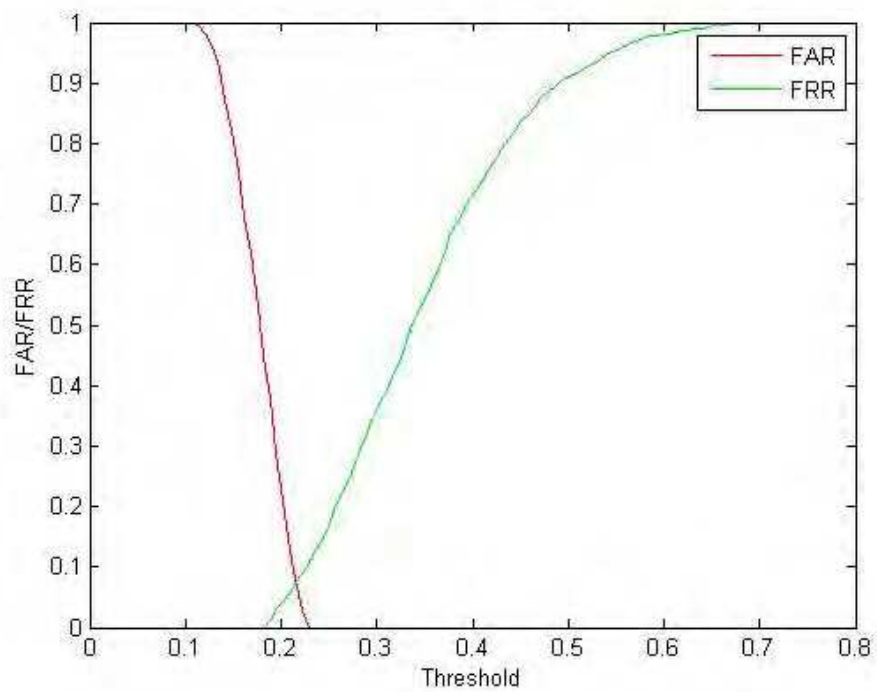

Fig.9 The FAR/FRR curves for various threshold values of the MoM. The intersection point corresponds to the ERR.

\section{Conclusion}

In this chapter, a SOM-based algorithm for fingerprint identification is presented. The minutiae of the template image are used as neurons of a neural network and the proposed algorithm detects the set of minutiae in the input image in an iterative way. The main advantage of this method, against other minutiae-based fingerprint recognition methods, is that the minutiae of only the template image have to be estimated. Furthermore the method is error-tolerant in the estimation of the minutiae of the template image. This is a matter of high importance since the precise estimation of minutiae is a difficult task, especially for low-quality fingerprint images. The overall performance of the proposed method was $92 \%$. It should be noted that the focus of the chapter was to show the feasibility of the SOM theory for registering fingerprint images. The proposed implementation of the SOM model could be considered as a method for finding the optimum value of an objective function. Under this framework, the proposed registration scheme provides several "degrees of freedom" regarding its parameters. For example, another measure of match (such as mutual information) could be used, other characteristic points of the fingerprint images (such as the points with high value of deviation) could be used, minutiae could be extracted by means of other methods. Moreover the algorithm has not been tested on partial fingerprint images or images with high degree of distortion. 


\section{References}

Bazen A. \& Gerez, S. (2002).Systematic Methods for the Computation of the Directional Fields and Singular Points of Fingerprints, IEEE Transactions on Pattern Analysis and Machine Intelligence, Vol. 24, Issue 7, Jul. 2002,pp. 905-919,ISSN:0162-8828.

Chan, K. C. ; Moon, Y. S. \& Cheng P.S. (2004). Fast Fingerprint Verification Using Subregions of Fingerprint Images, IEEE Transactions on Circuits and Systems For Video Technology., Vol.14, Issue 1, Jan. 2004,pp. 95-101,ISSN:1051-8215.

Chen, X.; Tian, J. \& Yang, X. (2006). A New Algorithm for Distorted Fingerprints Matching Based on Normalized Fuzzy Similarity Measure, IEEE Transactions on Image Processing, Vol. 15, Issue 3, March 2006,pp. 767-776, ISSN: 1057-7149

Gu, J.; Zhou, J. \& Yang, Ch.(2006). Fingerprint Recognition by Combining Global Structure and Local Cues, IEEE Transactions on Image Processing, Vol.15, Issue 7, Jul. 2006,pp.1952-1964, ISSN: 1057-7149.

Guo,Z.; \& Hall,R; Parallel Thinning with Two-Subiteration Algorithms,Communications, Association of Computer Machinery New York USA,Vol. 32 Issue 3,pp. 359373,1989,ISSN:0001-0782.

Hastings,R.(2007). Ridge Enhancement in Fingerprint Images Using Oriented Diffusion, Proceedings of the $9^{\text {th }}$ Biennial Conference of the Australian Pattern Recognition Society on Digital Image Computing Techniques and Applications, pp.245-252, ISBN: 0-7695-3067-2, Dec. 2007,IEEE Computer Society, Washington DC-USA

He, Y. ; Tian, J. ; Li, L. ; Chen, H. \& Yang, X. (2006). Fingerprint Matching Based on Global Comprehensive Similarity, IEEE Transactions on Pattern Analysis and Machine Intelligence, Vol. 28, Issue 6, Jun. 2006,pp. 850-862 ISSN:0162-8828.

Hong, L.; Wan,Y. \& Jain,A. Fingerprint Image Enhancement: Algorithm and Performance Evaluation, IEEE Transactions on Pattern Analysis and Machine Intelligence, Vol. 20, Issue 8, Aug. 1998,pp. 777-789,ISSN: 0162-8828.

Jain A., Hong, L. \& Bolle, R.(1997). On - Line Fingerprint Verification, IEEE Trans Pattern Analysis and Machine Intelligence, Vol. 19, Issue 4, Apr. 1997,pp. 302-314,ISSN: 0162 8828.

Jain, A.; Prabhakar, S.; Hong, L. \& Pankanti, Sh.(2000). Filterbank-Based Fingerprint Matching, IEEE Transactions on Image Processing, Vol. 9,Issue 5, May 2000,pp. 846859.

Jain, A.K; Chen, Y. \& Demirkus, M. (2007). Pores and Ridges: High-Resolution Fingerprint Matching Using Level 3 Features, IEEE Transactions on Pattert Analysis and Machine Intelligence, Vol.29, Issue 1, Jan.2007,pp. 15-27.

Jea, T-Y. \& Govindaraju, V. (2005). A minutia-based partial fingerprint recognition system. Elsevier Pattern Recognition, Vol. 38, Issue 10, Oct. 2005 pp. 1672- 1684

Kohonen,T.(2000). Self-Organizing Maps, 3rd Edition., Springer-Verlag, ISBN:3540679219, Berlin, Germany.

Liu, L. ; Jiang, T.; Yang,J. \& Zhu, Ch.(2006) Fingerprint Registration by Maximization of Mutual Information, IEEE Transactions on Image Processing, Vol. 15, Issue 5, May 2006,pp.1100-1110,ISSN:1057-7149.

Maltoni,D.; Maio,D; Jain,A.K. \& Prabhakar,S(2009) “Handbook of Fingerprint Recognition,Second Edition”, Springer, ISBN:978-1-84882-253-5,London. 
Matsopoulos,G.K; Asvestas,P.A; Mouravliansky,N.A. \& Delibasis,K.K.(2004) Multimodal registration of retinal images using Self Organizing Maps, IEEE Transactions on Medical Imaging, Vol. 23, Issue 12, Dec. 2004,pp. 1557-1563, ISSN:0278-0062.

Pratt, W.(2001), Digital Image Processing, Third Edition, Wiley-Interscience Publication, ISBN: 0-471-37407-5, California.

Press, W.H.; Teukolsky, S.A.; Vetterling, W.T. \& Flannery, B.P.(1992) Numerical Recipes in C: The Art of Scientific Computing $2^{\text {nd }}$ edition, Cambridge Univ. Press, ISBN : 0521437148,Cambridge U.K.

Ross A.; Dass, S. \& Jain, A.(2006) Fingerprint Warping Using Ridge Curve Correspondences, IEEE Transactions on Pattern Analysis and Machine Intelligence, Vol. 28, Issue 1, Jan. 2006, pp. 19-30,ISSN:0162-8828.

Thai R. (2003) ,Fingerprint Image Enhancement and Minutiae Extraction, Honours Thesis, School of Computer Science \& Software Engineering University of Western Australia,Australia

Tico,M. \& Kuosmanen,P.(2000) An algorithm for fingerprint image postprocessing. Proceedings of the Thirty-Fourth Asilomar Conference on Signals, Systems and Computers , Vol.2,pp. 1735-1739, ISBN : 0-7803-6514-3,Pacific Grove CA USA, Nov. 2000.IEEE

Website of NEUROtechnology,Biomedical and Artificial Technologies, http://www.neurotechnologija.com/download.html

Yager, N \& Amin, A. (2004). "Fingerprint classification: a review". Springer-Verlag Pattern Analysis E Applications, Vol. 7, Issue 1, April 2004,pp. 77-93,ISSN 1433-7541. 


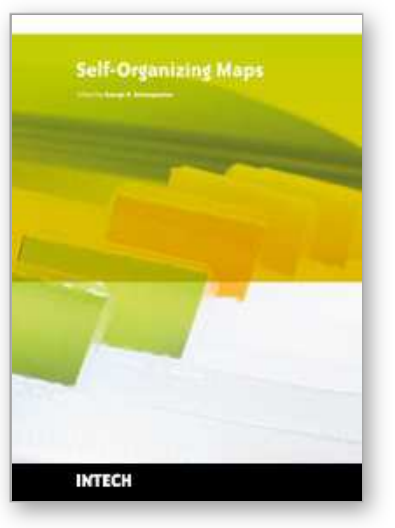

\author{
Self-Organizing Maps \\ Edited by George K Matsopoulos
}

ISBN 978-953-307-074-2

Hard cover, 430 pages

Publisher InTech

Published online 01, April, 2010

Published in print edition April, 2010

The Self-Organizing Map (SOM) is a neural network algorithm, which uses a competitive learning technique to train itself in an unsupervised manner. SOMs are different from other artificial neural networks in the sense that they use a neighborhood function to preserve the topological properties of the input space and they have been used to create an ordered representation of multi-dimensional data which simplifies complexity and reveals meaningful relationships. Prof. T. Kohonen in the early 1980s first established the relevant theory and explored possible applications of SOMs. Since then, a number of theoretical and practical applications of SOMs have been reported including clustering, prediction, data representation, classification, visualization, etc. This book was prompted by the desire to bring together some of the more recent theoretical and practical developments on SOMs and to provide the background for future developments in promising directions. The book comprises of 25 Chapters which can be categorized into three broad areas: methodology, visualization and practical applications.

\title{
How to reference
}

In order to correctly reference this scholarly work, feel free to copy and paste the following:

Anastasia N. Ouzounoglou, Pantelis A. Asvestas and George K. Matsopoulos (2010). Fingerprint Matching with Self Organizing Maps, Self-Organizing Maps, George K Matsopoulos (Ed.), ISBN: 978-953-307-074-2, InTech, Available from: http://www.intechopen.com/books/self-organizing-maps/fingerprint-matching-with-selforganizing-maps

\section{INTECH}

open science | open minds

\section{InTech Europe}

University Campus STeP Ri

Slavka Krautzeka 83/A

51000 Rijeka, Croatia

Phone: +385 (51) 770447

Fax: +385 (51) 686166

www.intechopen.com

\section{InTech China}

Unit 405, Office Block, Hotel Equatorial Shanghai

No.65, Yan An Road (West), Shanghai, 200040, China

中国上海市延安西路65号上海国际贵都大饭店办公楼405单元

Phone: +86-21-62489820

Fax: +86-21-62489821 
(C) 2010 The Author(s). Licensee IntechOpen. This chapter is distributed under the terms of the Creative Commons Attribution-NonCommercialShareAlike-3.0 License, which permits use, distribution and reproduction for non-commercial purposes, provided the original is properly cited and derivative works building on this content are distributed under the same license. 